\title{
Corticotropin-releasing Factor Changes the Phenotype and Function of Dendritic Cells in Mouse Mesenteric Lymph Nodes
}

\author{
Li Meng, Zhang Lu, Wang Xiaoteng, Hu Yue, Lu Bin, ${ }^{*}$ Meng Lina, and Chen Zhe \\ Department of Gastroenterology, First Affiliated Hospital of Zhejiang Chinese Medical University, Hangzhou, China
}

\section{Background/Aims}

Dendritic cells (DCs) are a significant contributor to the pathology of numerous chronic inflammatory autoimmune disorders; however, the effects of Corticotropin-releasing factor (CRF) on intestinal DCs are poorly understood. In this study, we investigated the role of CRF in alterations of intestinal dendritic cell phenotype and function.

\section{Methods}

Mouse mesenteric lymph node dendritic cells (MLNDCs) were obtained using magnetic bead sorting. Surface expression of CRF receptor type 1 (CRF-R1) and CRF-R2 was determined by double-labeling immunofluorescence and quantitative polymerase chain reaction (qPCR) and MLNDCs were subsequently exposed to CRF in the presence or absence of CRF-R1 and CRF-R2 antagonists. Expression of surface molecules (MHC-I and MHC-II) and co-stimulatory molecules (CD80 and CD86) was determined by flow cytometric and western blot analyses, and the T cell stimulatory capacity of MLNDCs was evaluated by mixed lymphocyte reaction.

\section{Results}

Immunofluorescent staining and quatitative polymerase chain reaction indicated that both the CRF receptors (CRF-R1 and CRF-2) are expressed on the surface of MLNDCs. Exposure to CRF increased the expression of MHC-II on MLNDCs as well as their capacity to stimulate T cell proliferation. MLNDCs treated with CRF-R1 antagonist exhibited a phenotype characterized by a less activated state and reduced surface expression of MHC-II, and consequently showed reduced capacity to stimulate T cells. In contrast, treatment of MLNDCs with CRF-R2 antagonist yielded an opposite result.

\section{Conclusions}

CRF can alter the phenotype and function of intestinal DCs through direct action on CRF-R1 and CRF-R2, and activation of the CRF-R1 and CRF-R2 pathways yields opposing outcomes.

(J Neurogastroenterol Motil 2015;21:571-580)

Key Words

Corticotropin-releasing hormone; Dendritic cells; Immunity, cellular

Received: January 30, 2015 Revised: June 11, 2015 Accepted: June 30, 2015

(c) This is an Open Access article distributed under the terms of the Creative Commons Attribution Non-Commercial License (http://creativecommons. org/licenses/by-nc/4.0) which permits unrestricted non-commercial use, distribution, and reproduction in any medium, provided the original work is properly cited.

*Correspondence: Lu Bin, MD

Department of Gastroenterology, First Affiliated Hospital of Zhejiang Chinese Medical University, 54 Youdian Road, Hangzhou 310006, China

Tel: +86-571-8703-2028, Fax: +86-571-8707-7785, E-mail: Ivbin@medmail.com.cn

Li Meng and Zhang Lu contributed equally to this work.

Financial support: This study was supported by Natural Science Foundation of China (Grant No. 81170348); Natural Science Foundation of Zhejiang province (Grant No. LZ12H03001); and Scientific research fund for the doctoral program of college (Grant No. 20113322110001).

Conflicts of interest: None.

Author contributions: Lu Bin designed and supervised the study; Li Meng, Wang Xiaoteng, Hu Yue, and Zhang Lu performed the majority of the experiments, wrote the manuscript, and surveyed; Meng Lina and Chen Zhe analyzed and interpreted data as well as reviewed the manuscript. All authors read and approved the final version to be published.

ORCID: Lu Bin, http://orcid.org /0000-0002-3381-6976, Li Meng, http://orcid.org /0000-0001-8921-2533. 


\section{Introduction}

Corticotropin-releasing factor (CRF), a 41-amino acid peptide produced largely in the paraventricular nucleus of the hypothalamus, is a major activator of the hypothalamo-pituitary-adrenal (HPA) axis. ${ }^{1,2}$ In addition, many studies have identified CRF expression in close proximity to $\mathrm{CRF}$ receptors in the colon, suggesting the existence of local autocrine or paracrine regulatory loops in the gastrointestinal (GI) tract. Consistent with the exquisite sensitivity of the GI tract, increasing evidence suggests that stress-related alterations of GI functions are mediated primarily through activation of CRF signaling pathways. ${ }^{3,4}$ Indeed, the GI dysmotility and visceral hypersensitivity induced by stress, which is quite common in irritable bowel syndrome (IBS) patients, are thought to play vital roles in the pathogenesis of IBS and can be relieved by the administration of CRF receptor antagonists.

The activity of CRF is mediated through surface receptors, known as CRF receptor type 1 (CRF-R1) and CRF-R2. Expression of CRF receptors was initially thought to be restricted to the brain and the pituitary, but was later shown to be widely distributed in the peripheral tissues and organs, including the heart and gut. ${ }^{4,6}$ In diseases of the digestive tract, studies have shown that CRF-R1 is the primary receptor involved in stress-induced alterations of lower gut secretomotor and pain sensation, whereas CRF-R2 activation inhibits the stress-induced colonic motor responses. ${ }^{7,8}$ Based on our previous findings, we postulate that CRF may have a significant role in the pathogenesis of IBS, specifically in the development of visceral hypersensitivity, through the opposing functions of CRF-R1 and CRF-R2.'

The intestinal lumen contains an abundance of foreign antigens and intestinal dendritic cells (DCs) are believed to play a significant role in intestinal immune homeostasis. The DCs of the intestinal lamina propria that recognize foreign antigens in the intestine migrate to the draining mesenteric lymph nodes (MLNs), where they present antigen to $\mathrm{T}$ cells. ${ }^{10}$ Recently, CRF-R expression in monocyte-derived DC (MoDC) has been reported, and it has also been suggested that CRF could modulate immune responses by acting directly on MoDCs. ${ }^{11}$ However, to date there are no reports of CRF-R expression on intestinal DCs and the relationship between $\mathrm{CRF}$ and intestinal DCs remains to be explored.

The objective of this study was to investigate the expression of CRF-R in mouse mesenteric DCs, as well as the relationship between CRF and intestinal DC function. We hypothesized that (1) both CRF-R1 and CRF-R2 are expressed on the surface of mesenteric lymph node dendritic cells (MLNDCs); and (2) that $\mathrm{CRF}$ could alter the phenotype and function of DCs through activation of distinct $\mathrm{CRF}$ receptors.

\section{Materials and Methods}

\section{Experimental Animals}

Male C57BL/6 mice weighing 15-20 g were housed under controlled conditions $\left(22 \pm 1^{\circ} \mathrm{C}, 65-70 \%\right.$ humidity) with a 12-hour light/dark cycle at the Animal Center of Zhejiang Chinese Medical University, Hangzhou, China; water and food were provided ad libitum. Experimental protocols conformed to the requirements of the State Authority for Animal Research Conduct.

\section{Isolation of Mesenteric Lymph Node Dendritic Cells}

Mice were euthanized by cervical dislocation and mesenteric lymph nodes from the ileocecal junction were harvested immediately under sterile conditions. Tissues were immersed in $5 \mathrm{~mL}$ RPMI1640 (Gibco) and agitated gently between frosted glass slides. Tissue preparations were passed through a $200-\mathrm{mm}$ cell filter to obtain single cell suspensions and cells were centrifuged twice at $200 \mathrm{~g}$ for 10 minutes each. DCs were then incubated with a mixture of CD11c microbeads (Miltenyi Biotec, Bergisch Gladbach, Germany), FcR blocking reagent (Miltenyi Biotec), and buffer in a $1: 1: 3$ ratios for 15 minutes at $4^{\circ} \mathrm{C}$. Following incubation, DC populations were enriched through magnetic bead sorting on MACS columns (Miltenyi Biotec); sorting was performed twice to obtain higher purity of DCs. The purity of dendritic cells based on surface phenotype of isolated cells was determined by flow cytometric analysis (FACSCantoII, BD Biosciences, San Jose, USA); typical purity of the cell population following magnetic sorting was approximately 70-80\% (CD11c positivity).

\section{Cell Cultures and Reagents}

Following purification, MLNDCs were cultured in RPMI1640 with $10 \%$ fetal bovine serum, $100 \mathrm{IU} / \mathrm{mL}$ penicillin and $100 \mathrm{ug} / \mathrm{mL}$ streptomycin and incubated at $37^{\circ} \mathrm{C}, 5 \% \mathrm{CO}_{2}$.

Reagents: phosphate buffered saline (PBS; GNM-20012, Hangzhou, China), RPMI1640 and 10\% fetal bovine serum 
(Gibco, Grand Island, NY, USA), PE-anti-mouse CD11c, APC-anti-mouse MHC-I, FITC-anti-mouse MHC-II, FITCanti-mouse CD80, APC-anti-mouse CD86, PE-mouse IgG1 control, APC-mouse IgG2a control, FITC-mouse IgG2b control (eBioscience, Sandiego, California, USA), 4\% paraformaldehyde (Sigma, St. Louis, Missouri, USA), blocking solution (Sigma), Anti-CRHR1 (Anbo Biotechnology, San Francisco, California, USA), Anti-CRHR2 (Anbo Biotechnology), FITC anti-Mouse CD11c (eBioscience), $\mathrm{Cy} 3$ conjugated goat anti-rabbit IgG (H+L), 4',6-diamidino-2-phenylindole (DAPI) (Southern Biotech Associates, Birmingham, Alabama, USA), anti-fading agent (Sigma). Ten percent of SDS-PAGE (Amersham Biosciences, Piscataway, New Jersey, USA), polyvinylidene fluoride (PVDF) membranes (Amersham Biosciences), goat anti-rabbit MHC-II (Abcam, Cambridge, UK), HRP-conjugated secondary antibody(Abcam). Carboxyfluorescein diacetate succinimidyl ester (Invitrogen Ltd, Paisley, UK), anti-mouse CD4 antibody, and anti-mouse CD8 antibody (Invitrogen Ltd).

\section{Morphological Observation of Dendritic Cells}

Five hundred microliters of unstained DC cell suspension was transferred to a glass slide and overlaid with a cover slip. Cell morphology was observed and images were obtained by COIC XDS-1B inverted optical microscope (COIC, Chongqing, China).

\section{Immunofluorescence for Corticotropin-releasing Factor Receptors}

Cytospins of samples were prepared by centrifugation at 1000 rpm for 5 minutes at $4^{\circ} \mathrm{C}$ (Wescor, Wrentham, Massachusetts, USA) and dried rapidly to prevent cell detachment from the coverslip. Cells were fixed in $4 \%$ paraformaldehyde/phosphate buffered saline (PBS)/0.1\% Triton-X100 for 10 minutes at room temperature and rinsed with PBS $(3 \times 5$ minutes $)$. Coverslips were blocked in $50-100 \mu \mathrm{L}$ blocking solution for 30 minutes at room temperature. After washing with PBS $(3 \times 5$ minutes $)$, sections were incubated in a humidified chamber with both CRF-R antibodies diluted to $1: 50$ and FITC anti-Mouse $\mathrm{CD} 11 \mathrm{c}$ diluted to $1: 20$ at $4^{\circ} \mathrm{C}$ overnight. Sections were washed with PBS $(3 \times 5$ minutes $)$ and incubated for 1 hour at room temperature in a humidified chamber with $\mathrm{Cy} 3$ conjugated goat anti-rabbit $\operatorname{IgG}(\mathrm{H}+\mathrm{L})$ diluted to 1:400, protected from light. After three rinses with PBS, samples were counterstained with DAPI for 15 minutes, then mounted onto slides and cover slipped with aqueous mounting medium containing anti-fading agent for immunofluorescence analysis.

Cell immunofluorescence was analyzed using BD Pathway435 High Content Bioimagers (BD Biosciences, Franklin Lakes, USA) and Nikon intensilight C-HGFI Fiber Illuminator (Nikon Corporation, Tokyo, Japan); digital images were acquired and adjusted using COMOS software.

\section{Detection of Corticotropin-releasing Factor Receptor mRNA by Quantitative Polymerase Chain Reaction}

Total RNA was extracted from DCs using an RNeasy Mini Kit (Roche, Basel, Switzerland) following the manufacturer's instructions. Reverse transcription reactions were performed using $1 \mu \mathrm{g}$ of total RNA. Amplification was performed on a GeneAmp PCR system (PTC0200; Bio-Rad Laboratories, Hercules, CA, USA). Qauntitative polymerase chain reaction $(\mathrm{qPCR})$ reactions were then run in duplicate, with each reaction containing $5 \mu \mathrm{L} 2 \times$ FastStart Universal SYBR Green Master mix, $0.2 \mu \mathrm{L}$ each target-specific primer, $0.2 \mu \mathrm{L}$ Rox reference dye, $1 \mu \mathrm{L}$ cDNA template, and $3.4 \mu \mathrm{L}$ PCR-grade water in a total $10 \mu \mathrm{L}$ reaction volume (Table). Cycling conditions were as follows: pre-denaturation at $95^{\circ} \mathrm{C}$ for 30 seconds, followed by 44 cycles consists of denaturation at $95^{\circ} \mathrm{C}$ for 5 seconds and anneal-

Table. Primer Sequences

\begin{tabular}{|c|c|c|c|}
\hline \multirow{2}{*}{ Chondrogenic marker } & \multirow{2}{*}{ Symbols } & \multicolumn{2}{|c|}{ mRNA Sequence } \\
\hline & & Forward primer & Reverse primer \\
\hline $\begin{array}{l}\text { Corticotropin-releasing factor type } 1 \text { receptor } \\
\text { (NM_007762) }\end{array}$ & CRF-R1 & TTTCTACGGTGTCCGCTAC & GGATGAAAGCCGAGATGAG \\
\hline $\begin{array}{l}\text { Corticotropin-releasing factor type } 2 \text { receptor } \\
\text { (NM_001288618) }\end{array}$ & CRF-R2 & ATCCTGATGACGAAGTTACGAG & GAAGAAGCAGTAGAAAACGGAC \\
\hline $\begin{array}{l}\text { Glyceraldehyde-3-Phosphate Dehydrogenase } \\
\text { (NM_001289726) }\end{array}$ & GAPDH & AGAAGGTGGTGAAGCAGGCATC & CGAAGGTGGAAGAGTGGGAGTTG \\
\hline
\end{tabular}

CRF-R, corticotropin-releasing factor receptor; GAPDH, glyceraldehyde-3-phosphate dehydrogenase (house keeping gene). 
ing at $60^{\circ} \mathrm{C}$ for 30 seconds, extension at $72^{\circ} \mathrm{C}$ for 30 seconds, then $72^{\circ} \mathrm{C}$ for 7 minutes, and $4^{\circ} \mathrm{C}$ forever.

The extracted DNA was assayed with $1.2 \%$ agarose gels (High Strength Analytical Grade Agarose; Bio-Rad Laboratories) in TBE buffer (50 nM Tris, $50 \mathrm{nM}$ borate, $1.25 \mathrm{nM}$ EDTA, $\mathrm{pH}$ 8.2), stained with ethidium bromide for 30 minutes at $60 \mathrm{~mA}$ and photographed under UV light. A 100 bp to 500 bp DNA ladders were used as a molecular size marker.

\section{Identification of Cell Surface Markers by Flow Cytometry}

Freshly isolated MLNDCs were plated in 96-well plates $\left(1 \times 10^{5}\right.$ cells per well), and pretreated with the CRF-R1 antagonist antalarmin $(10 \mathrm{nM})$ or the CRF-R2 antagonist astressin 2B $(10 \mathrm{nM})$ for 30 minutes prior to incubation with CRF $(10 \mathrm{nM})$. After 24 hours, expression of MLNDCs surface markers was determined by flow cytometry. Briefly, cell were collected and washed, resuspended in $\mathrm{PBS}$ and stained with the following mixtures of antibodies: MHC-I/APC, MHC-II/FITC, CD86/APC, CD80/FITC, PE-mouse IgG1 control, APC-mouse IgG2a control, and FITC-mouse IgG2b control, all at manufacturer's recommended concentrations. After incubation for 30 minutes at $4^{\circ} \mathrm{C}$, cells were washed and centrifuged at $1500 \mathrm{rpm}$ for $5 \mathrm{mi}-$ nutes and resuspended in PBS. Fluorescence was recorded using a FACS Calibur and samples were analyzed using FACSDIVA software (BD Biosciences).

\section{Western Blot Analysis of Cell Surface Markers}

DCs were prepared for Western blot analysis as described above for flow cytometry in order to determine the expression of MHC-II. Cell lysates were resolved using 10\% SDS-PAGE and transferred onto PVDF membranes. Membranes were blocked with $5 \%$ nonfat milk in PBS containing $0.1 \%$ Tween-20 (Sigma) at $4^{\circ} \mathrm{C}$ overnight with gentle rocking and probed with primary antibodies at $4^{\circ} \mathrm{C}$ (goat anti-rabbit MHC-II; diluted to 1:1200). Following washing, the membrane was incubated with HRPconjugated secondary antibody (1:10 000 dilution) for 2 hours at room temperature, and visualized using an ECL detection kit (Amersham Biosciences) and X-ray film.

\section{Mixed Leukocyte Reactions}

MLNDCs were seeded into 96-well plates at $5 \times 10^{5}$ cells/well and incubated with OVA (10 ng/mL) for 2 hours. MLNDCs were pretreated for 30 minutes with antalarmin $(10 \mathrm{nM})$ or astressin $2 \mathrm{~B}$ (10 $\mathrm{nM})$, prior to exposure to CRF (10 nM).

Single cell suspensions derived from spleens of male C57BL/6 mice, were labelled with carboxyfluorescein diacetate succinimidyl ester (CFSE) for assessment of $\mathrm{T}$ cell proliferation. ${ }^{12}$ Equal numbers of CFSE-labeled lymphocytes were added to each well and cultured for 7 days in RPMI 1640 medium containing $10 \%$ heat-inactivated fetal calf serum, $2 \mathrm{mM}$ L-glutamine, 100 units $/ \mathrm{mL}$ penicillin, and $100 \mu \mathrm{g} / \mathrm{mL}$ streptomycin. Cells were then removed from the wells and stained with anti-mouse CD4 or CD8 antibody, respectively. Proliferation of gated CFSE-labeled CD4/CD8-positive responder $\mathrm{T}$ cells was evaluated via CFSE attenuation by flow cytometry using the CFSE Geometric Mean Fluorescence Intensity.

\section{Statistical Methods}

Data were expressed as mean $\pm \mathrm{SD}$; statistical differences in cell surface expression of CD80, CD86, MHC-II, and MHC-I were compared using the independent samples t test after determining that groups exhibited normal distributions and equal variance. Statistical analyses were performed using SPSS17.0 software; $P<0.05$ was considered statistically significant.

\section{Results}

\section{Morphological Observation of Mesenteric Lymph Node Dendritic Cells}

Freshly sorted MLNDCs were round, small cells exhibiting protrusions of various lengths (Fig. 1).

\section{Expression of Corticotropin-releasing Factor} Receptor Type 1 and Corticotropin-releasing Factor Receptor Type 2 on the Surface of Mesenteric Lymph Node Dendritic Cells

The expression of CRF-R proteins on MLNDCs was confirmed by immunofluorescence staining and qPCR (Fig. 2). Mouse hypothalamus was included as a positive control in the result of qPCR.

\section{Effects of Corticotropin-releasing Factor Stimulation on Expression of Dendritic Cell Surface Molecules}

In order to examine the effect of CRF on expression of MLNDC surface molecules cells were exposed to CRF $(10 \mathrm{nM})$ for 24 hours, and the expression of CD86, CD80, MHC-II, and 

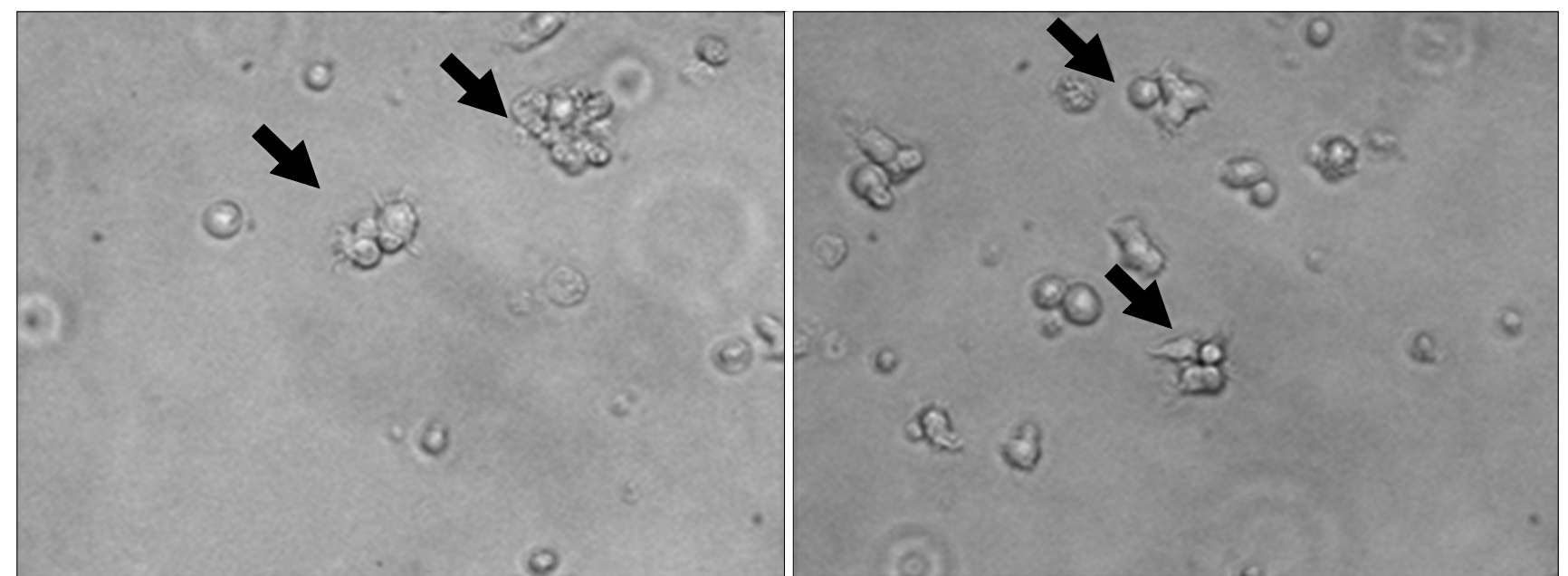

Figure 1. Mesenteric lymph node dendritic cell (MLNDC) morphology. MLNDCs have a stellate shape, with large and long sheet-like processes extending in multiple directions from the cell body. These arrows point to typical dendritic branches of MLNDCs (magnification, $\times 400$ ).

A
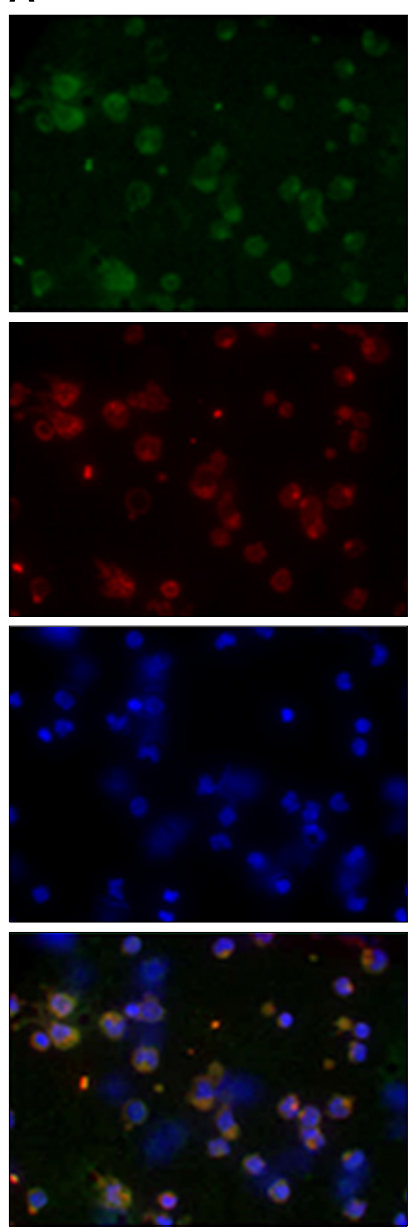
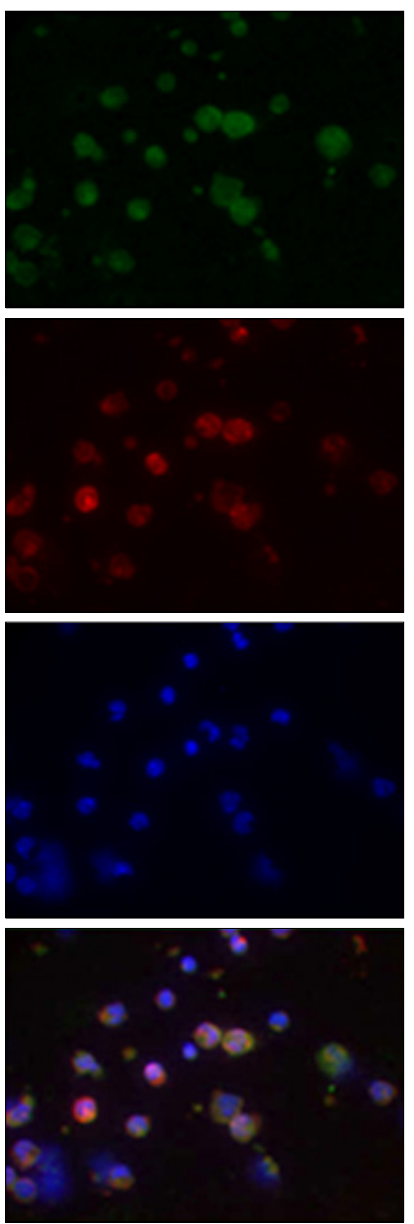

B Positive CRF-R1 Marker CRF-R2 Positive

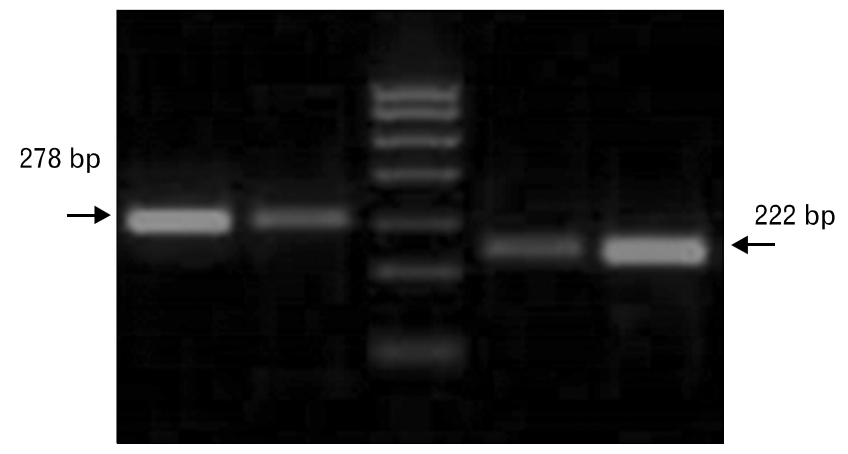

Figure 2. The expression of corticotropin-releasing factor receptor type 1 (CRF-R1) and CRF-R2 on the surface of mesenteric lymph node dendritic cells (MLNDCs). (A) Co-immunofluorescence staining and confocal micrscopic analysis of MLNDCs using antibodies against CD11C (green), CRF-R (red), and DAPI counterstaining (blue). CRF-R1 (left), CRF-R2 (right), FITC, green Fluoresceinisothiocyanate, $\mathrm{Cy} 3$, indocarbocyanin red and DAPI 4',6-Diamidino-2- phenylindoldihydrochlorid blue-nuclear counterstaining (magnification, $\times 600$ ). (B) The expression of CRF-R on mouse MLNDCs and hypothalamus was confirmed by quantitative polymerase chain reaction. CRF-R1 (size 278 bp) and CRF-R2 (size 222 bp) were identified with the help of positive group (mouse hypothalamus). 

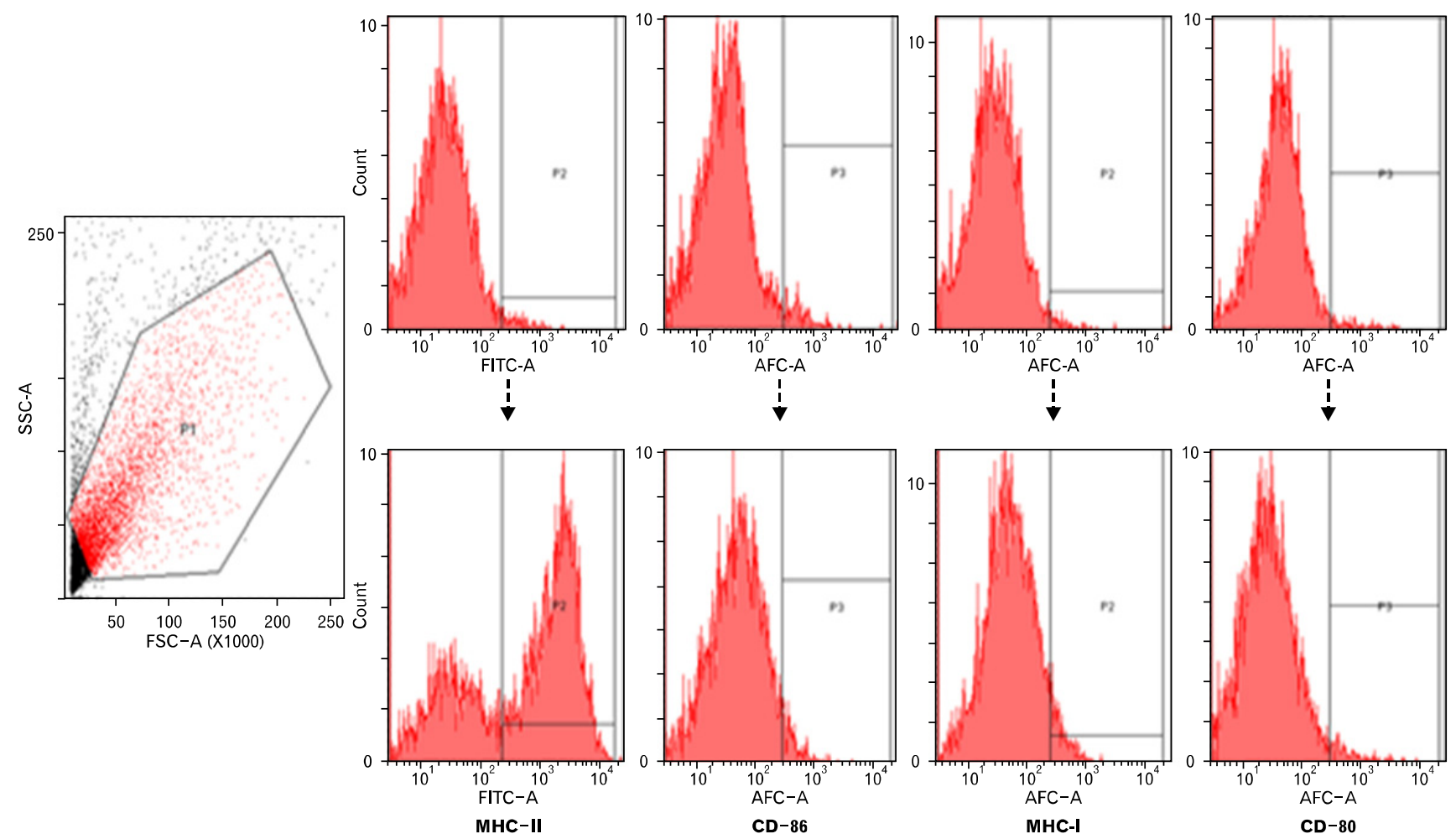

Figure 3. The expression of surface molecules of mesenteric lymph node dendritic cells (MLNDCs). The result of flow cytometry shows high level of MHC-II was expressed on the surface of MLNDCs, whereas the expression of CD80, CD86, and MHC-I was low.

MHC-I was determined by flow cytometry and western blot analyses. Incubation of MLNDCs with CRF (10 nM) enhanced the expression of MHC-II $(P<0.05)$; in contrast, the expression of CD80, CD86, and MHC-I was unchanged (Fig. 3). To confirm that this effect was mediated via CRF receptors, MLNDCs were pretreated for 30 minutes with the selective CRF-R1 antagonist antalarmin $(10 \mathrm{nM})$ prior to incubation with CRF (10 nM). Results indicated decreased expression of MHC-II with antalarmin pretreatment compared to $\mathrm{CRF}$ alone $(P<0.05)$. Moreover, pretreatment of MLNDCs for $30 \mathrm{mi}-$ nutes with the selective CRF-R2 antagonist astressin 2B (10 $\mathrm{nM})$ prior to incubation with $\mathrm{CRF}(10 \mathrm{nM})$ resulted in markedly increased expression of MHC-II compared to CRF treatment alone $(P<0.05)$ (Fig. 4).

\section{Corticotropin-releasing Factor Stimulated T Lymphocytes Proliferation in Mesenteric Lymph Node Dendritic Cells}

Consistent with the ability of mature DCs to stimulate proliferation of T lymphocytes, MLNDCs cocultured with CRF $(10 \mathrm{nM})$ induced significantly higher proliferation of $\mathrm{T}$ cells compared with the control group. Additionally, the pro-proliferative effect induced by CRF was enhanced by astressin $2 \mathrm{~B}$ pre-treatment, while antalarmin yielded opposite effects (Fig. 5).

\section{Discussion}

The results from our study demonstrate that both CRF-R1 and CRF-R2 are expressed on the surface of MLNDCs in mice and that CRF can alter the phenotype and function of DCs through increased expression of surface molecules as well as their capacity to stimulate $\mathrm{T}$ cells. To our knowledge, this is the first study to investigate a relationship between CRF expression and MLNDC function.

CRF mediates its effects via $2 \mathrm{G}$-protein-coupled receptors (CRF-R1 and CRF-R2) and plays important neuromodulatory roles in stress responses and synaptic plasticity. In the gut, CRF-R1 is primarily distributed in the myenteric neuronal plexus, submucosal neuronal plexus, and the lamina propria of the colonic mucosa, ${ }^{13,14}$ whereas CRF-R2 is expressed mainly on blood vessels of the submucosal layer and in the intestinal mucosa, located in the luminal surface of crypts. DCs act as a link between 
A
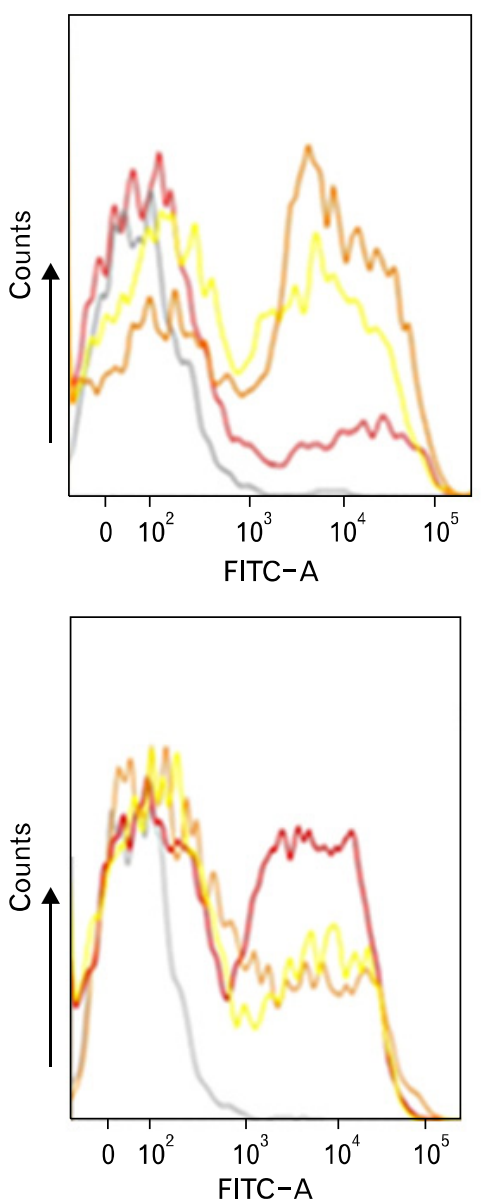

B

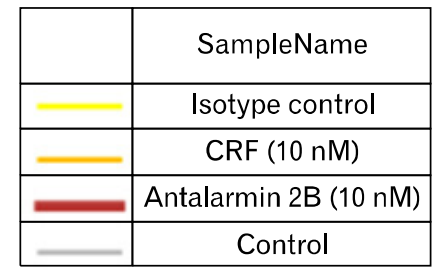

C

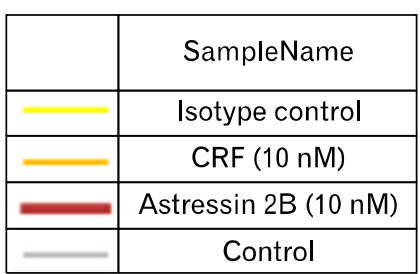

Figure 4. Effects of corticotropin-releasing factor (CRF) stimulation on dendritic cell (DC) surface molecule expression. (A) The result of flow cytometry shows enhanced expression of MHC-II when incubated with CRF and decreased expression with corticotropin-releasing factor receptor type 1 (CRF-R1) antagonist antalarmin $(10 \mathrm{nM})$ pretreatment compared to CRF alone. Moreover, pretreatment of mesenteric lymph node dendritic cells (MLNDCs) with CRF-R2 antagonist astressin 2B (10 nM) resulted in markedly increased expression of MHC-II compared to CRF treatment alone. (B) Western blot analysis of MHC-II expression using the anti-MHC-II antibody in MLNDCs incubated with CRF after 30 minute pretreatment with the CRF-R1 antagonist antalarmin (10 nM) or the CRF-R2 antagonist astressin 2B (10 nM); glyceraldehyde-3-phosphate dehydrogenase (GAPDH) expression was used as a loading control. MHC-II expression was upregulated when incubated with CRF and pretreated with a CRF-R2 antagonist, but downregulated when pretreated with a CRF-R1 antagonist. (C) Bar graph showing quantitation of MHC-II expression in (B).

the innate and adaptive immune systems, and Lee et $\mathrm{al}^{11}$ reported that CRF-R1 and CRF-R2 were detected in human MoDC. However, CRF-R expression in MLNDCs has not been previously described, and we report here for the first time that both CRF-R1 and R2 proteins are present in MLNDCs. Our findings have important implications for biological roles for CRF in MLNDCs.

As highly specialized professional antigen-presenting cells, dendritic cells initiate and regulate pathogen-specific adaptive immune responses by presenting processed peptides to $\mathrm{T}$ cells and native antigens to $\mathrm{B}$ cells. ${ }^{9}$ In the gut, classical DCs are the main antigen presenting cells, characterized by high cytokine producing capacity as mature cells. Classical DCs can be found in intestinal lamina propria, Peyer's patches, and intestinal draining lymph nodes, including the MLNs. Evidence indicate that DCs may be critical for stress associated diseases such as IBS, ${ }^{15}$ and our previous work indicates that MLNDCs play a significant role in the development of visceral hypersensitivity in IBS by mediating mast cell activation. ${ }^{16}$ Interestingly, there is a growing body of research focused on understanding the relationship between CRF and immune system alterations on molecular and cellular levels, as well as in vivo. Experimental evidence suggests 
A
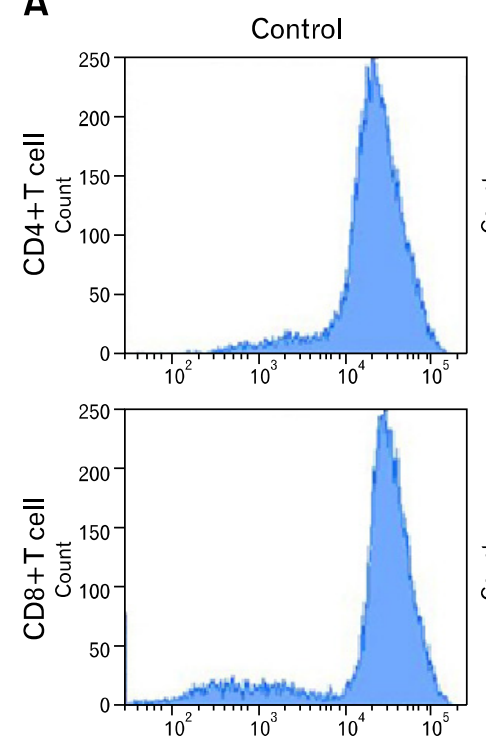

B
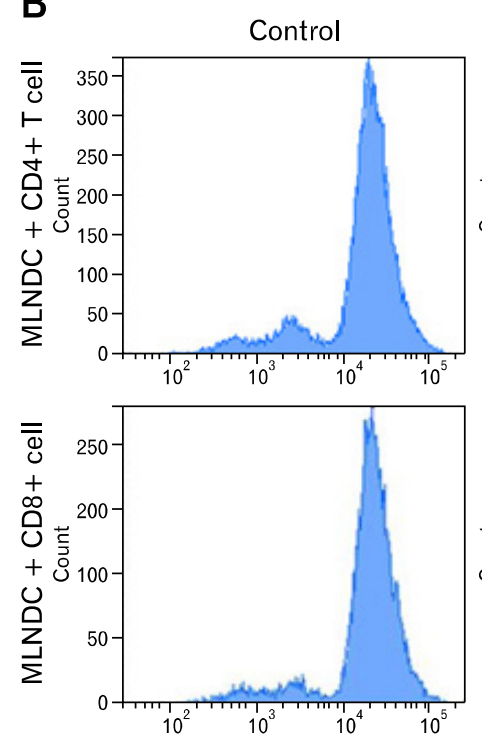

$\mathrm{DC}: \mathrm{T}=1: 1$
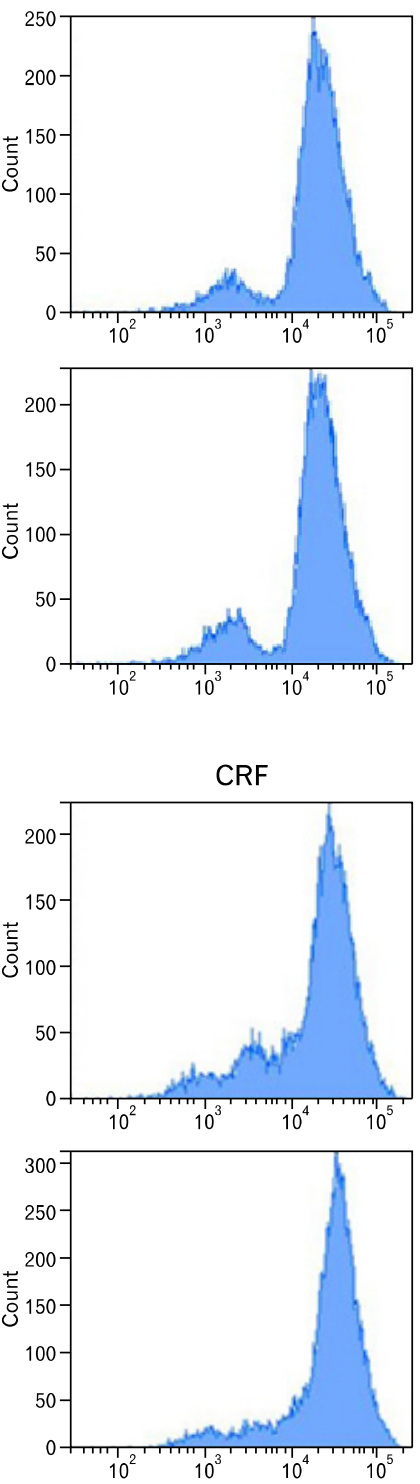
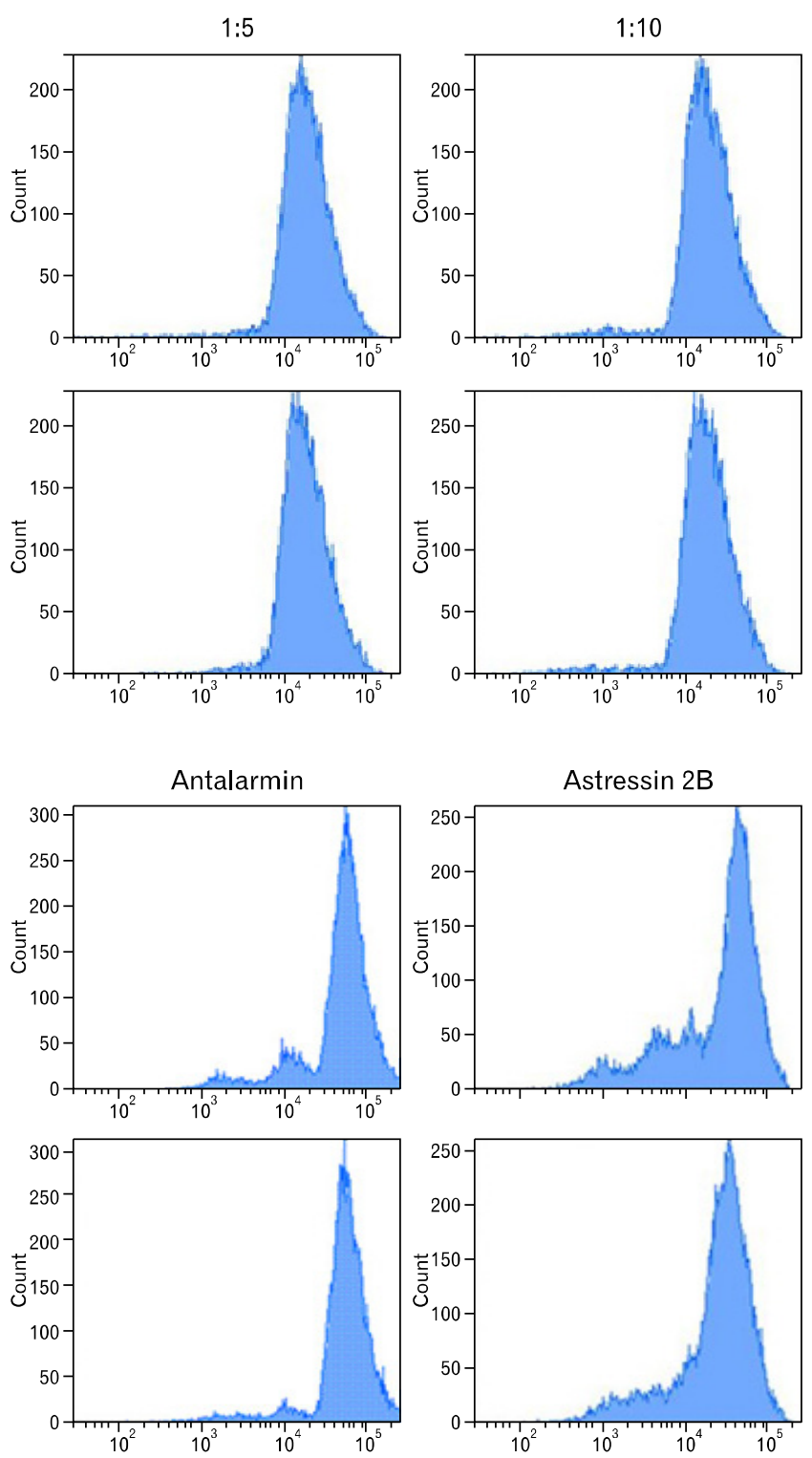

Figure 5. Corticotropin-releasing factor $(\mathrm{CRF})$ treatment increases $\mathrm{T}$ lymphocyte stimulatory capacity of mesenteric lymph node dendritic cells (MLNDCs). (A) MLNDCs $\left(5 \times 10^{5}\right)$ were cultured for 7 days with increasing numbers of carboxyfluorescein diacetate succinimidyl ester (CFSE)-labeled T cells from spleens of C57BL/6 mice. Representative CFSE profiles of CD4+/CD8 + T cells are shown. DC:T indicates the ratio of DC to T cells. (B) Representative CFSE profiles of CD4+/CD8 + T cells after 7 days in culture under various conditions. Control, $\mathrm{CD} 4+/ \mathrm{CD} 8+$ T cells with MLNDCs; CRF, CD4+/CD8 + T cells with MLNDCs in the presence of CRF (10 nM); CRF-R1 antagonist, CD4+/CD8 + T cells with MLNDCs in the presence of CRF (10 nM), MLNDCs were pretreated with CRF-R1 antagonist antalarmin (10 nM) for 30 minutes; CRF-R2 antagonist, $\mathrm{CD} 4+/ \mathrm{CD} 8+\mathrm{T}$ cells plus MLNDCs in the presence of $\mathrm{CRF}(10 \mathrm{nM})$, MLNDCs were pretreated with CRF-R2 antagonist astressin $2 \mathrm{~B}(10 \mathrm{nM})$ for 30 minutes. Cells were cultured with $5 \times 10^{5} \mathrm{~T}$ cells, $5 \times 10^{5} \mathrm{MLNDCs}$.

that the cellular components of the immune-mediated inflammatory response, such as mast cells, monocytes-macrophages, as well as lymphocytes T, B, and natural killer, are closely related to CRF. ${ }^{17-21}$ Until now, it had only been reported that CRF could regulate the expression of IL-18 in MoDC, ${ }^{11}$ and this study is the first to demonstrate a functional role for CRF in MLNDCs. We observed increased levels of MHC-II after culturing MLNDCs with CRF, indicating that CRF could promote MLNDC maturation. We further showed that CRF increases the ability of MLNDCs to stimulate CD4+ and CD8 $+\mathrm{T}$ 
lymphocytes. Since DCs are critical in the context of early immune responses, our results suggest that that CRF has the potential to modulate immune responses by acting directly on MLNDCs.

In the GI tract, activation of peripheral CRF-R1 has been shown to play a key role in the processes of increased intestinal permeability and visceral hypersensitivity. ${ }^{22,23}$ In contrast, activation of CRF-R2 by administration of CRF-R2 agonist can prevent visceral pain induced by colorectal distention. ${ }^{24} \mathrm{With}$ respect to the relationship between these two receptors, it has been reported that CRF-R1 is the primary receptor involved in stress-induced alterations of lower gut secretomotor and pain sensations, whereas CRF-R2 regulates the CRF-R1-mediated stimulation of intestinal motor function. Interestingly, experimental studies suggest that $\mathrm{CRF}$ could mediate mucosal barrier dysfunction in human colonic mucosa via CRF-R1/R2 receptors expressed on subepithelial mast cells. ${ }^{25}$ Additionally, pathway-specific effects of CRF-R1/R2 in cell survival and chemokine activation on monocytes have been identified. ${ }^{26}$ In this work, we describe novel, differential roles of $\mathrm{CRF}$ receptor subtypes in the phenotype and function of MLNDCs. Based on analysis using CRF receptor antagonists, we find that the CRF-R1 pathway enhances the expression of antigen-presenting molecules, consistent with increased capacity to stimulate T cells, while these effects are significantly inhibited by pretreatment with CRF-R2. These results demonstrate that CRF acts directly on CRF-R1 and CRF-R2 expressed on MLNDCs, resulting in the modulation of activation of MLNDCs as well as their T cell stimulatory functions.

Alterations in intestinal DC function may contribute to dysregulated immune responses which underlie chronic inflammatory disorders such as Crohn's disease and ulcerative colitis. Based on our previous study as well as recent reports, it is believed that DC function is altered during the development of the stress-related disorder IBS. On basis of our experimental observations, we conclude that CRF alters the phenotype and function of intestinal DCs through its 2 receptors. In response to activation of CRF-R1 and CRF-R2, DCs may induce distinct immune responses to various commensal bacteria, food antigens, and self-antigens, leading to alterations in visceral sensitivity and intestinal motility, ultimately resulting in distinct impacts on the development of IBS. Furthermore, drugs that block either CRF-R1 or CRF-R2 receptors may modify immune responses and may be considered for the treatment for disorders associated with CRF.

DCs are a functionally heterogeneous population which can be classified into different subtypes based on different surface markers. CD11c is a relatively common biomarker for three kinds of DC populations, eg, the plasmocytoid (pDC), the conventional lymphoid (cDC-lymphoid), and the conventional myeloid (cDC-myeloid). ${ }^{27}$ Moreover, CD11c is expressed on all defined DC subsets in mice and CD11c microbeads are used to isolate highly pure DCs from spleen and lymph nodes, Peyer's patches, and colonic lamina propria. ${ }^{28-30}$ However, we have to admit that CD11c is not a specific marker of DCs. The more detailed MLNDCs characterization with other DCs markers will help us better understand the effect of CRF on MLNDCs, and which is the main limitation of our study. Besides, recently, there have been reports of autocrine effects of CRF on dendritic cells. ${ }^{31,32}$ It had been proved that commensal bacteria such as Fusobacterium varium and Bacteroides vulgatus could augment the production of CRF in human MoDCs as well as JAWSII cells, a mouse dendritic cell line, which indicated the potential role of CRF produced by DCs themselves. Take it together with our current findings, it seemed that the immunomodulatory role for CRF by peripheral DCs may due to autocrine and/or paracrine interactions. However, whether the CRF could be released by MLNDCs themselves and its ability to alter the phenotype and function of intestinal DCs need further investigation.

In conclusion, we demonstrate that CRF could alter the phenotype and function of intestinal DCs through its actions on CRF-R1 and R2, and that these 2 CRF-Rs exert opposite effects in the formation of IBS. Although we have begun to investigate the relationship between CRF and intestinal DCs, further studies are required to elucidate the precise mechanisms of abnormal immune responses mediate by DCs in the development of stress-related diseases.

\section{References}

1. Chrousos GP. The hypothalamic-pituitary-adrenal axis and immune-mediated inflammation. N Engl J Med 1995;332:1351-1363.

2. Hillhouse EW, Grammatopoulos DK. The molecular mechanisms underlying the regulation of the biological activity of corticotropin releasing hormone receptors: implications for physiology and pathophysiology. Endocr Rev 2006;27:260-286.

3. Stengel A, Taché Y. Neuroendocrine control of the gut during stress: corticotropin-releasing factor signaling pathways in the spotlight. Annu Rev Physiol 2009;71:219-239.

4. Taché Y, Bonaz B. Corticotropin-releasing factor receptors and stress-related alterations of gut motor function. J Clin Invest 2007; 117:33-40.

5. Sagami Y, Shimada Y, Tayama J, et al. Effect of a corticotropin- releasing hormone receptor antagonist on colonic sensory and motor function in patients with irritable bowel syndrome. Gut 2004;53: 958-964. 
6. Wu SV, Yuan PQ, Wang L, Peng YL, Chen CY, Taché Y. Identification and characterization of multiple corticotropin-releasing factor type 2 receptor isoforms in the rat esophagus. Endocrinology 2007;148:1675-1687.

7. Gourcerol G, Wu SV, Yuan PQ, et al. Activation of corticotropin-releasing factor receptor 2 mediates the colonic motor coping response to acute stress in rodents. Gastroenterology 2011;140:15861596.

8. Taché Y, Million M. Role of Corticotropin-releasing factor signaling in stress-related alterations of colonic motility and hyperalgesia. $\mathrm{J}$ Neurogastroenterol Motil 2015;21:8-24.

9. Zhou H, Lü B, Zhang L, et al. The effect of antagonizing corticotropin releasing factor receptor 1 /activating corticotropin releasing factor receptor 2 on visceral sensitivity and colonic motility of irritable bowel syndrome rats. Chin J Dig 2011;31:372-373.

10. Coombes JL, Powrie F. Dendritic cells in intestinal immune regulation. Nat Rev Immunol 2008;8:435-446.

11. Lee HJ, Kwon YS, Park CO, et al. Corticotropin-releasing factor decreases IL-18 in the monocyte-derived dendritic cell. Exp Dermatol 2009;18:199-204.

12. Bernardo D, Al-Hassi HO, Mann ER, et al. T-cell proliferation and forkhead box $\mathrm{P} 3$ expression in human $\mathrm{T}$ cells are dependent on T-cell density: physics of a confined space? Hum Immunol 2012;73: 223-231.

13. Chatzaki E, Crowe PD, Wang L, Million M, Taché Y, Grigoriadis DE. CRF receptor type 1 and 2 expression and anatomical distribution in the rat colon. J Neurochem 2004;90:309-316.

14. Yuan PQ, Million M, Wu SV, Rivier J, Taché Y. Peripheral corticotropin releasing factor (CRF) and a novel CRF1 receptor agonist, stressin1-A activate CRF1 receptor expressing cholinergic and nitrergic myenteric neurons selectively in the colon of conscious rats. Neurogastroenterol Motil 2007;19:923-936.

15. Long Y, Wang W, Wang H, Hao L, Qian W, Hou X. Characteristics of intestinal lamina propria dendritic cells in a mouse model of postinfectious irritable bowel syndrome. J Gastroenterol Hepatol 2012;27:935-944.

16. Li M, Zhang L, Lü B, Meng LN, Chen Z, Chu L. Abnormal dendritic cells mediated immune response in a rat model of visceral hypersensitivity. Zhonghua Yi Xue Za Zhi 2013;93:2904-2908.

17. Kempuraj D, Papadopoulou NG, Lytinas M, et al. Corticotropinreleasing hormone and its structurally related urocortin are synthesized and secreted by human mast cells. Endocrinology 2004;145: 43-48.

18. Radulovic M, Dautzenberg FM, Sydow S, Radulovic J, Spiess J. Corticotropin-releasing factor receptor 1 in mouse spleen: expression after immune stimulation and identification of receptor-bearing cells. J Immunol 1999;162:3013-3021.

19. Wang W, Zhang X, Yang L, Liu D, Liu G, Zhou J. Lipopolysac- charide upregulates the expression of corticotropin-releasing hormone via MAP kinase pathway in rat peritoneal macrophages. Mol Cell Biochem 2012;361:1-7.

20. Singh VK, Fudenberg HH. Binding of $\left[{ }^{125} \mathrm{I}\right]$ corticotropin releasing factor to blood immunocytes and its reduction in Alzheimer's disease. Immunol Lett 1988;18:5-8.

21. Boyadjieva N, Advis JP, Sarkar DK. Role of beta-endorphin, corticotropin-releasing hormone, and autonomic nervous system in mediation of the effect of chronic ethanol on natural killer cell cytolytic activity. Alcohol Clin Exp Res 2006;30:1761-1767.

22. Larauche M, Gourcerol G, Wang L, et al. Cortagine, a CRF1 agonist, induces stresslike alterations of colonic function and visceral hypersensitivity in rodents primarily through peripheral pathways. Am J Physiol Gastrointest Liver Physiol 2009;297:G215-G227.

23. Rodiño-Janeiro BK, Alonso-Cotoner C, Pigrau M, Lobo B, Vicario $\mathrm{M}$, Santos J. Role of corticotropin-releasing factor in gastrointestinal permeability. J Neurogastroenterol Motil 2015;21:33-50.

24. Million M, Wang L, Wang $\mathrm{Y}$, et al. CRF2 receptor activation prevents colorectal distension induced visceral pain and spinal ERK1/2 phosphorylation in rats. Gut 2006;55:172-181.

25. Wallon C, Yang PC, Keita AV, et al. Corticotropin-releasing hormone (CRH) regulates macromolecular permeability via mast cells in normal human colonic biopsies in vitro. Gut 2008;57:50-58.

26. Chandras C, Koutmani Y, Kokkotou E, Pothoulakis C, Karalis KP. Activation of phosphatidylinositol 3-kinase/protein kinase B by corticotropin-releasing factor in human monocytes. Endocrinology 2009; 150:4606-4614

27. Li J, Park J, Foss D, Goldschneider I. Thymus-homing peripheral dendritic cells constitute two of the three major subsets of dendritic cells in the steady-state thymus. J Exp Med 2009;206:607-622.

28. Williamson E, Bilsborough JM, Viney JL. Regulation of mucosal dendritic cell function by receptor activator of NF-kappa B (RANK)/ RANK ligand interactions: impact on tolerance induction. J Immunol 2002;169:3606-3612.

29. Ing R, Segura M, Thawani N, Tam M, Stevenson MM. Interaction of mouse dendritic cells and malaria-infected erythrocytes: uptake, maturation, and antigen presentation. J Immunol 2006;176:441-450.

30. Krajina T, Leithäuser F, Möller P, Trobonjaca Z, Reimann J. Colonic lamina propria dendritic cells in mice with $\mathrm{CD} 4+\mathrm{T}$ cell-induced colitis. Eur J Immunol 2003;33:1073-1083.

31. Koido S, Ohkusa T, Kan S, et al. Production of corticotropin-releasing factor and urocortin from human monocyte-derived dendritic cells is stimulated by commensal bacteria in intestine. World J Gastroenterol 2014;20:14420-14429.

32. Hojo M, Ohkusa T, Tomeoku H, et al. Corticotropin-releasing factor secretion from dendritic cells stimulated by commensal bacteria. World J Gastroenterol 2011;17:4017-4022. 